\title{
Article
}

\section{A Coupled Euler-Lagrange CFD Modelling of Droplets-To-Film}

\author{
Adeniyi, Akinola, Morvan, Herv'e P. and Simmons, Kathy A \\ Available at http://clok.uclan.ac.uk/19963/ \\ Adeniyi, Akinola ORCID: 0000-0003-0768-9341, Morvan, Herv'e P. and \\ Simmons, Kathy A (2017) A Coupled Euler-Lagrange CFD Modelling of \\ Droplets-To-Film. The Aeronautical Journal, 121 (1246). pp. 1897-1918. ISSN \\ 0001-9240
}

It is advisable to refer to the publisher's version if you intend to cite from the work. http://dx.doi.org/10.1017/aer.2017.107

For more information about UCLan's research in this area go to

http://www.uclan.ac.uk/researchgroups/ and search for <name of research Group>.

For information about Research generally at UCLan please go to http://www.uclan.ac.uk/research/

All outputs in CLoK are protected by Intellectual Property Rights law, including Copyright law. Copyright, IPR and Moral Rights for the works on this site are retained by the individual authors and/or other copyright owners. Terms and conditions for use of this material are defined in the policies page.

\section{CLoK}

Central Lancashire online Knowledge www.clok.uclan.ac.uk

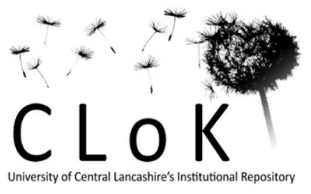




\title{
A Coupled Euler-Lagrange CFD Modelling of Droplets-To-Film
}

\author{
Akinola A. Adeniyi* \\ aadeniyi@uclan.ac.uk \\ Motorsports \& and Mechanical Engineering \\ School of Engineering \\ University of Central Lancashire \\ Preston \\ United Kingdom, PR1 2HE
}

Hervé P. Morvan and Kathy A. Simmons

Gas Turbine \& Transmissions Research Centre (G2TRC)

University of Nottingham

Nottingham

United Kingdom, NG7 2RD

\section{ABSTRACT}

In this paper, a droplet to film interaction model technique is presented. In the proposed approach, the liquid and gas continua are modelled using an enhanced Volume of Fluid (VoF) technique while the droplets are tracked using a Lagrangian framework and are coupled to the Eulerian phases using source terms. The eventual target application is an aeroengine bearing chamber in which oil is found as droplets, shed from the bearings, splashing on impact, separated from wall surfaces at obstacles or simply re-entrained, and as a continuum oil film coating the bearing chamber outer walls which it also cools. In finite volume CFD techniques, a prohibitively large number of cells would be required to describe the details of the droplet impact phenomenon. Based on published correlations, the splashing droplets are created and tracked as Lagrangian particles. The flowing film and the gas continua are handled with an enhanced Volume of Fluid technique.

${ }^{*}$ Address correspondence to this author. 


\section{NOMENCLATURE}

\begin{tabular}{|c|c|}
\hline$A, B$ & Constants \\
\hline$\vec{A}, \vec{B}$ & Arbitrary vectors \\
\hline CFL & Courant-Friedrichs-Lewy \\
\hline$C_{D}$ & Coefficient of drag \\
\hline DPM & Discrete Particle Model \\
\hline$D_{p}$ & Droplet diameter \\
\hline$E_{m}$ & Mass fraction of splashed droplets relative to the impacting droplet \\
\hline$F$ & Force \\
\hline$F_{d}, F_{n}, f$ & Droplet train frequency \\
\hline$F_{D}$ & Drag force \\
\hline$F_{s}$ & Surface tension force \\
\hline$g$ & Gas phase,Gravity \\
\hline$h^{*}$ & Relative film thickness \\
\hline$h$ & Grid spacing \\
\hline$h_{f i l m}, R_{w}$ & Film thickness \\
\hline$H_{x}^{*}, H_{f}^{*}$ & Geometry dimension relative droplet diameter \\
\hline$H_{\epsilon}$ & Heaviside function \\
\hline$H_{x}, H_{y}, H_{z}$ & Geometry dimension \\
\hline$K$ & Splash factor \\
\hline La & Laplace number \\
\hline$\dot{m}_{p}$ & mass flow rate of droplet \\
\hline$M$ & Inter-phase mass exchange \\
\hline$M_{p}$ & Mass of a droplet particle before impact \\
\hline$M_{s}$ & Mass of a droplet particle splashing \\
\hline$N_{s}$ & Number of splashing droplets \\
\hline$N_{x}, N_{y}, N_{z}, N_{f}$ & Number of nodes \\
\hline $\mathrm{Oh}$ & Ohnesorge number \\
\hline$p$ & pressure \\
\hline$P$ & 3D spatial position \\
\hline $\operatorname{Re}$ & Reynolds number \\
\hline$R_{s}, R$ & Actual radius occupied by a droplet continuum \\
\hline$S, S_{\alpha}, S_{m}$ & Source terms \\
\hline$S^{*}$ & Droplet size relative to grid spacing \\
\hline$t$ & flow time \\
\hline$U, u$ & Velocity (continuum) \\
\hline$U_{p}, U_{o}$ & Droplet velocity \\
\hline$V_{\text {cell }}$ & Volume of a computational cell \\
\hline VoF & Volume of Fluid \\
\hline$W e$ & Weber number \\
\hline$X_{c}$ & Centroid of a cell \\
\hline$X_{p}$ & Particle position \\
\hline
\end{tabular}




\section{Greek Symbol}

$v \quad$ Kinematic viscosity

$\sigma \quad$ Surface tension

$\rho \quad$ Density

$\mu \quad$ Dynamic viscosity

$\alpha \quad$ Volume fraction

$\Lambda \quad$ A fluid property

$\phi \quad$ Level set function

$\kappa \quad$ Curvature

$\delta, \delta_{z} \quad$ Delta function

$\epsilon \quad$ Very thin layer

$\Delta t \quad$ flow time step

\section{Subscripts}

$l \quad$ Liquid phase

$m \quad$ Mass

$o \quad$ Before impact 


\subsection{Introduction}

In specialised engineering applications, liquids can be encountered in different forms; as well formed droplets, or as a well defined continuum such as film on wall or as sheet or jets of the liquid. The morphology of the liquid is determined by the interaction of the gas phase and physical properties such as surface tension and viscosity. Oil, for example, is used for lubrication and cooling. Atomising the oil provides a large volume to surface area ratio to convey a high heat flux. In internal combustion engines atomised engine oil is sprayed to the cylinder walls below the piston area to lubricate the piston-cylinder rubbing. In aeroengines, oil is used to lubricate high speed ball bearings. In this type of application, the oil becomes atomised as it leaves the bearings ${ }^{(1)}$. In these applications, it is easy to visualise that the atomised oils eventually form a continuum after impact. The continuous oil film can be collected for a reuse. This work is motivated from such applications that involve droplet interaction with and formation of a continuous film. The ability to model such interaction efficiently is therefore key and a CFD model to do so is presented in this paper.

Droplets can also be formed by stripping off from the free liquid surface as a result of Raleigh-Taylor instabilities ${ }^{(2)}$ or by thin film navigating a sharp bend ${ }^{(3)}$ or from ligament breakup ${ }^{(4)}$. Upon impact of droplets on a dry or pre-wetted wall, secondary droplets (splash) can be formed depending on the impact parameters ${ }^{(5)}$. Droplet to film interaction is of great engineering interest and has been widely researched, starting with Reynolds ${ }^{(6)}$ experiment in 1875 , although, overall only a few impact scenarios, such as impact on a gentle film, have been investigated. Other relevant scenarios such as the oblique angle droplet impact on arbitrary moving film on a surface or filaments suspended in space are still lacking. This area of research remains very active aided with advances in camera technology and a wide areas of application to technologies ${ }^{(7)}$. Modelling is getting more and more relevant for understanding a design. Upon impact of a droplet on a wetted or dry-surface, there are a number of possible outcomes respectively named stick, spread, rebound, or splash as described by Yarin ${ }^{(5)}$. The trampoline behaviour is a newer regime reported by Bisighini ${ }^{(6)}$ in which the free surface deforms like a trampoline after droplet impact. The normal-impact of single droplet on dry and pre-wetted surface was studied by ${ }^{(8-12)}$; others did impact of a drop train on a heated surface $^{(13)}$. There are only a few publications on oblique impact, where the droplet impact at some oblique angle to the surface such as in $\breve{S i k a l o ~ e t ~ a l ~}{ }^{(14)}$.

As the interest in droplet to film experiments is still on-going, so is the modelling of this phenomenon. The free-interface tracking Volume-of-Fluid (VoF) based Computational Fluid Dynamics (CFD) techniques has been successfully used to model both the droplet and the film by fully resolving each droplet onto a CFD mesh grid such as done in Riever \& Frohn ${ }^{(15)}$ and Peduto et al ${ }^{(16)}$, for example. The VoF model was originally proposed by Hirt \& Nichols ${ }^{(17)}$ for numerically treating fluid boundaries embedded in a computational cell. It is a relatively affordable way of dealing with segregated flows. VoF is indeed a homogenous Eulerian approach that assumes a single fluid continuum of varying properties defined by a so called "colour functions", typically the volume fractions. It is common to define the interface as the iso-surface of volume fraction of $50 \%$. Using the volume fraction to define the segregated interface between fluid regions is fine for most computations.

Using the volume fraction in the computation of the surface curvature, however, in the commonly used Brackbill surface tension model ${ }^{(18)}$ can result in over-prediction of the surface tension force. This can for example lead to unphysical dry outs ${ }^{(19)}$. Osher \& Sethian ${ }^{(20)}$ have defined the interface using a signed continuous Level Set (LS) function. The free inter- 
face is here defined as the zero iso-surface of the LS function. The Level Set (LS) function ${ }^{(21)}$ provides a better interface curvature prediction although LS on its own possesses poor mass conservation ${ }^{(22,23)}$. Bourlioux ${ }^{(24)}$ was first to propose the coupling of both Level Set and VoF, otherwise called Coupled Level Set Volume of Fluid (CLSVoF). The CLSVoF technique serves to correct for such over-prediction in surface tension. For example in flows past obstacles and where surface tension is significant ${ }^{(25)}$.

It is possible to simulate the impact and the post-impact outcome of droplets interacting with walls and film using VoF techniques by fully resolving the flow using very fine mesh elements but this can be computationally very expensive; for example, Peduto et al ${ }^{(16)}$ used over 3 million computational cells to resolve a single droplet splash. In a multiple droplet impact scenario fully resolving the droplets to the mesh grid is computationally expensive and may not be practical for a complex engineering configuration. Alternative techniques that are computationally affordable are therefore desirable. A method proposed in this work is to employ the Lagrangian tracking technique to the droplets, whereas, the film and gas phases are treated using the Eulerian VoF technique. Such approach is also known as the Discrete Particle Model or DPM in ANSYS-Fluent which is used to support the work presented in this paper. The two-way interaction between the DPM droplets and the gas phase is achieved by coupling the Lagrangian and Eulerian phases through drag force terms. The coupling of the 3 numerical components (air, oil-film $\mathcal{E}$ oil-droplet), and in particular the (liquid) droplets with the liquid film and or filaments, is achieved by managing source terms in the governing equations. This method requires free-surface tracking and also to determine when a liquid droplet (DPM) is going to interact with the liquid phase in VoF. Existing works ${ }^{(15,16)}$ have shown that without using a very fine mesh it is impossible to resolve such phenomenon as droplet splash. From experimental observations, ${ }^{10,26,27)}$, correlations exist to characterise the splashing of droplets. Conventionally, the primary droplet is the droplet approaching the film and the splashing droplets are often referred to as children or secondary droplets. Correlations from such works inform the creation of child droplet products, where applicable.

The commercial solver, ANSYS-Fluent 14.5 is employed in the present work. The interaction between DPM and Eulerian-VoF is available in ANSYS-Fluent but coupling of the DPM (which is physically the liquid phase) to the liquid component of VoF, to the authors knowledge and as proposed here, is new. The technique is numerically checked for mass and momentum conservation. Momentum conservation is checked using the crater evolution of a single drop impact on a film against simulation and correlations from literature ${ }^{(10,15,28,29)}$. For ease of computation, the film thickness, $h^{*}$, has been used to describe dry walls $\left(h^{*}=0\right)$, thin film $\left(0<h^{*}<1\right)$, intermediate film $\left(h^{*}=1\right)$ and deep pools $\left(h^{*}>>1\right)$. In this work, a Lagrangian representation is also proposed to continue tracking splashing secondary droplets where experimental correlations are available for such impact regime.

\subsection{Formulating the DPM-CLSVoF method}

The impact sequence assumes that the droplet is firstly in the gas phase and travels towards a wall or a film. The droplet hits the film interface or a dry wall. At low impact energies, the droplets become part of the film and do not create extra child droplets. At high energy impact, child droplets are formed after absorbing part of the primary droplet. We should be able to predict the free film interface, track the primary droplet in the gas phase and its impact onto the film, as well as create the splashed children numerically. 
The Navier-Stokes and continuity equations formulation for incompressible flow, respectively, given in Eq. (1) and (2) are employed. The Navier-Stokes equations represent the force balance at each point in the domain. The inertial terms are balanced with the divergence of stress, body forces and other contributory source terms such as $\overrightarrow{S_{m}}$.

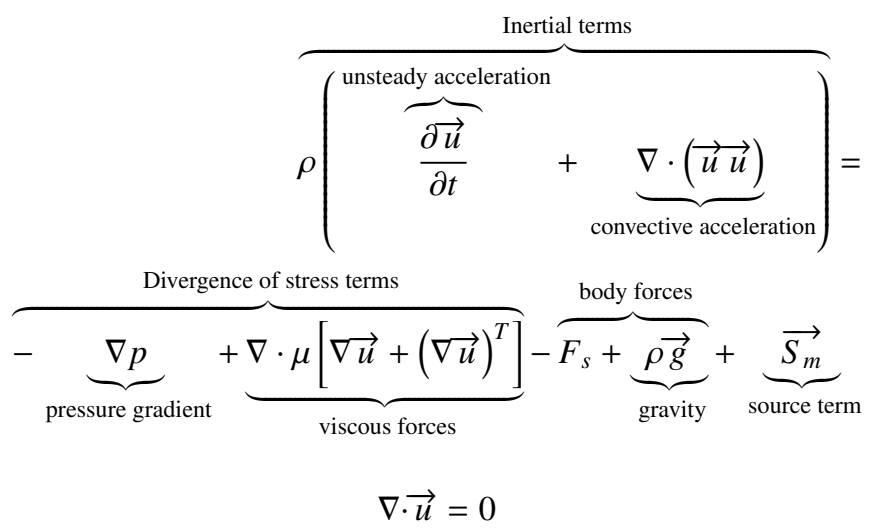

where $F_{s}$, is the surface tension force added as a spatially varying body force and only near the free surface.

\subsection{Coupled Level Set VoF (CLSVoF)}

The VoF technique is essentially solving the continuity equation for the volume fraction of the present phases in the form of Eq. (3).

$$
\frac{\partial}{\partial t}\left(\alpha_{i} \rho_{i}\right)+\nabla \cdot\left(\alpha_{i} \rho_{i} u_{i}\right)=S_{\alpha_{i}}+M_{n e t}
$$

where $M_{n e t}$ is the net mass exchange within the phases such as may obtain during evaporation or condensation. Physical phase change is not modelled in this work, thus $M_{n e t}$ is zero. $S_{\alpha_{i}}$ is the source term. The primary phase volume fraction is not computed using Eq. (3), but rather restricted to conserve mass using Eq. (4). For a liquid and gas multiphase flow, where the air phase is taken as the primary phase, for example, $\alpha_{l}=1-\alpha_{g}$.

$$
\sum_{i=1}^{n} \alpha_{i}=1
$$

The Level Set (LS) function, $\phi$, is advected using Eq. (5). The Level Set function, $\phi$, can take positive and negative values; the interface is described at the zero Level Set and the liquid phase is described with positive Level Set function values.

$$
\frac{\partial \phi}{\partial t}+u \cdot \nabla \phi=0
$$

The equation will move the zero Level Set exactly as the free-surface motion from the initially known position of the film. The Level Set function is smooth and continuous; this makes it possible to accurately compute the spatial gradients. This capability of the Level Set 
method provides a good estimation of the interface curvature, Eq. (6) and in turn the surface tension force, Eq. (7), required in the Navier-Stokes Equation (1).

$$
\begin{gathered}
\kappa(\phi)=\left.\nabla \cdot \frac{\nabla \phi}{|\nabla \phi|}\right|_{\phi=0} \\
F_{s}=\sigma \kappa(\phi) \nabla \phi \delta_{\varepsilon}(\phi)
\end{gathered}
$$

where $\delta_{\varepsilon}$ [Eqn. 8] is a delta function, smoothed over a distance $\varepsilon$, this is by assuming that the interface can be considered as a "thin" fluid region with a thickness $\varepsilon$.

$$
\delta_{\varepsilon}(\phi)= \begin{cases}\frac{1}{2 \varepsilon}\left(1+\cos \left(\frac{\pi \phi}{\varepsilon}\right)\right) & \text { if }|\phi|<\varepsilon \\ 0 & \text { otherwise }\end{cases}
$$

where $\varepsilon$ is taken as 1.5 times the grid spacing.

In Level Set method ${ }^{(21)}$, the effective density and viscosity of the fluid are calculated using Eqs. (9a) \& (9b) respectively.

$$
\begin{aligned}
& \rho=\rho_{g}+\left(\rho_{l}-\rho_{g}\right) H_{\varepsilon}(\phi) \\
& \mu=\mu_{g}+\left(\mu_{l}-\mu_{g}\right) H_{\varepsilon}(\phi)
\end{aligned}
$$

where $H_{\varepsilon}(\phi)$ is a smoothed Heaviside function.

$$
H_{\varepsilon}(\phi)= \begin{cases}0 & \text { if } \phi<-\varepsilon \\ \frac{(\phi+\varepsilon)}{(2 \varepsilon)}+\frac{1}{(2 \pi)} \sin \left(\frac{\pi \phi}{\varepsilon}\right) & \text { if }|\phi| \leq \varepsilon \\ 1 & \text { if } \phi>\varepsilon\end{cases}
$$

The level set function is smooth and continuous; this makes it possible to accurately compute its spatial gradients. This capability of the level set method provides a good estimation of the interface curvature and in turn the surface tension force in Eq. (1). Level set alone is, however, not mass conservative. In the VoF formulation, there is discontinuity at the free-surface posing a weakness for the method to compute the spatial gradients. A major strength of the VoF method is its mass conservation ability. The coupling of both level set and the volume of fluid (CLSVoF) methods ensures mass conservation, the ability to capture the free-surface and to correctly compute surface tension force.

In this work, the standard CLSVOF coupling in ANSYS-Fluent ${ }^{(30)}$ has been implemented without modification to the advection techniques. In the partially filled cells (i.e. cells containing both gas and liquid) where the coupling is required (Fig. 1), the reconstruction of the interface is done using both LS and VoF. Basically, the VoF model predicts the fraction of the cell to be sliced $\left(y_{1}-y_{1}\right)$ by the free-surface. The gradient of the LS function $(\phi)$ is used to predict the direction $\left(N_{1}\right)$ of the free surface. The computational approach of CLSVoF is similar to those of those of piecewise linear interface construction (PLIC) ${ }^{(23)}$. It involves the reconstruction of the free-surface front and a minimisation of the front points to the interface (Fig. 2).

The algorithm for coupling LS and VoF in ANSYS-Fluent ${ }^{(30)}$ basically determines partially filled cells using either the LS or VoF criteria. A free-surface is located where the signs of $\phi$ alternate or where $0<\alpha<1$. The normal $\left(N_{2}\right)$ of the free-surface in the cell segment are calculated using the gradient of $\phi$. In positioning the free-interface "slicing" of cells, at 


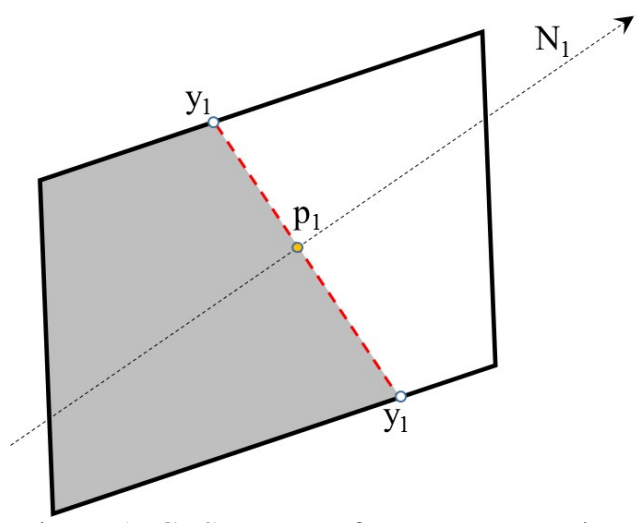

Figure 1: CLSVoF Interface Reconstruction I

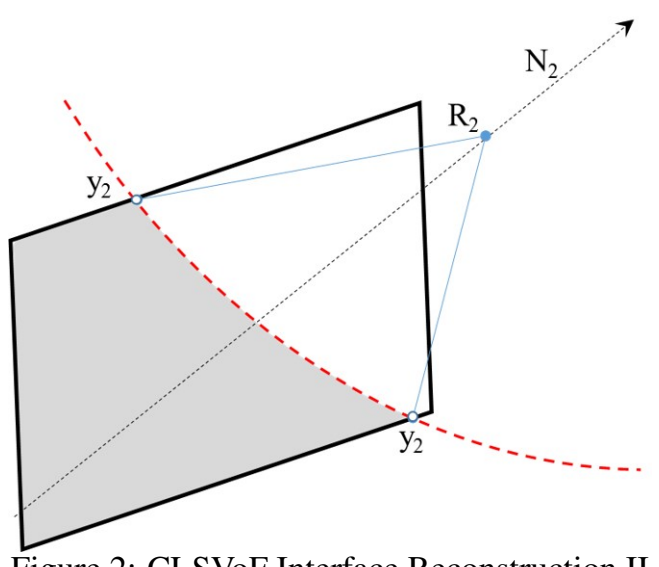

Figure 2: CLSVoF Interface Reconstruction II

least one corner of the cell is ensured to be occupied with the liquid phase relative to the neighbouring cells. In a way to satisfy VoF, the intersection of the free-surface normal with cell centreline $\left(p_{1}\right)$ is determined. The intersection points, or the "front points" $\left(y_{1}\right)$, of the free-surface and the cell boundaries are determined. Having constructed these fronts, the distance of a reference point $\left(R_{2}\right)$ on the normal inside the control volume to the free-surface is minimised. If the distance passes through the sliced segment, this is taken as the minimum distance $\left(y_{2}\right)$. Otherwise, if it is beyond the end points of the slice, the shortest distance from the reference point to the end of the points of the sliced segment is taken as the minimum distance. All the possible distances from the reference point to all the front cut-segments are minimised to give the distance from the free-surface. The free-interface deforms and has uneven profile with a thickness across the interface (Eq. 5). This does not guarantee that $|\nabla \phi|=1$ is always maintained. Therefore, the level set function is initialised with these distances in the next time step. 


\subsection{DPM-gas phase}

Consider a flow comprising of a mixture of air and liquid. The liquid can exist as a flowing continuum and as tiny droplets. A droplet in this work is a liquid of the same property as the flowing liquid. When the droplet is entrained in the gas phase, it is assumed that it can be treated as if it were a solid sphere before it encounters a continuum of liquid or it impacts a wall. The coordinates of the centre, $P_{(x, y, z)}$ of a droplet in a 3D space are obtained from integrating the force per unit mass balance, Eq. (11). The actual space occupied by the diameter is considered negligible relative to the entire control volume, thus not resolved as a continuum.

$$
\frac{d \overrightarrow{U_{p}}}{d t}=\underbrace{\overrightarrow{F_{D}}}_{\text {drag }}+\underbrace{\overrightarrow{F_{\text {otherforces }}}}_{\text {pressure, virtual mass, bouyancy }}
$$

The Reynolds number of the particle, $R e$, is estimated as $R e \simeq \rho_{l} D_{p}\left|\vec{U}_{p}-\vec{U}\right| / \mu$. The coefficient of drag, $C_{D}$, is computed depending on the scenario and can be implemented as a User Define Function (UDF) in ANSYS-Fluent ${ }^{(30)}$.

$$
\overrightarrow{F_{D}}=\frac{18 \mu}{\rho_{l} D_{p}^{2}} \frac{C_{D} \operatorname{Re}}{24} \frac{\vec{U}_{p}-\vec{U}}{\left|U_{p}-U\right|}
$$

The droplets are assumed to be non-deforming and behave like hard spheres, an assumption made because of the small sizes of the droplets. The drag coefficient is computed based on the Reynolds number using the Schiller \& Naumann ${ }^{(31)}$ correlations as given in Eq. (13); this correlation is considered sufficient as the deformation of a droplet has not been considered in this work. Interference drag ${ }^{(32)}$ experienced by droplets trailing another are assumed negligible.

$$
C_{D}= \begin{cases}24 \frac{1}{R e}, & R e \leq 1 \\ 24 \frac{1}{R e}\left(1+0.15 R e^{0.68}\right) & 1<\operatorname{Re}<1000, \\ 0.45, & 1000 \leq \operatorname{Re} \leq 3500\end{cases}
$$

In the implementation of the Lagrangian phase representation of the droplet, when the Lagrangian droplets (DPM particles) do not affect the Eulerian phase but the continous Eulerian flow fields do affect the DPM particles, it is known as "One-Way Coupling". "Two-way Coupling" is achieved when both the DPM and the Eulerian phases affect each other. In the Two-way Coupling approach, the DPM solution iteration alternates between the Eulerian phase solution iteration until convergence is reached. When coupled, the momentum transfer into the Eulerian phase, for example as $S_{m}$ in Eq. (1), is expressed in the form given in Eq. 14.

$$
\vec{F}=\sum[\frac{18}{24} \frac{\mu C_{D} R e}{\rho_{l} D_{p}^{2}}\left(\vec{U}_{p}-\vec{U}\right)+\underbrace{\vec{F}_{\text {other }}}_{\text {other interaction forces }}] \dot{m}_{p} \Delta t
$$

where $\dot{m}_{p}$ is the mass flow rate of the particulate phase and $\Delta t$ is the time step. 


\subsection{Coupling DPM-liquid component}

Section $\$ 2.2$ gives the handling of the droplet in the gas-only phase. When the Lagrangian droplet particle coordinates crosses into a cell containing the flowing liquid phase or bounded by a wall from the air phase, the droplet particle is removed from the solution and converted to "liquid" VoF using source terms. After "removal" of the droplet particle, it is added to the VoF liquid using a mass source term.

\subsubsection{DPM-CLSVoF Mass Exchange}

As the droplet travels in the solution domain, it might be the case that the droplet diameter becomes, on some occasions, larger than the current cell dimensions, which needs to be dealt with. This implies that the mass of the droplet will not "fit" the cell, violating the DPM basic assumptions and the incompressibilty principle adopted in our approach. In situations where the droplet size is bigger than the cell, the mass source term is therefore distributed accross a "Spread Radius" zone, $R_{s}$, Fig. 3. This zone represents the actual spherical volume that would have been occupied by the droplet if exists as a continuum (in VoF). In the example shown, the particle size is larger than the current cell size.

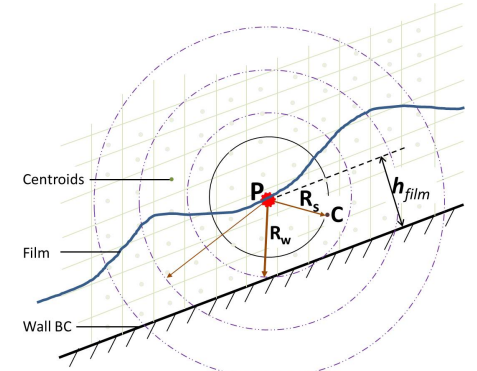

Figure 3: Scan zone: from impact point to the centroids

The mass source given by Eq.(15) is added to Eq. (3) only in the affected cells. The corresponding momentum source is given by Eq. (17) is also added in Eq. (1). The liquid source term represents the mass of liquid phase occupied in the zone. It should be noted that a mass of an equal volume of the gas needs to be taken out from the corresponding zone. This can be achieved using Eq. (16).

$$
S_{\alpha}=\frac{\rho_{i}}{\rho_{l}} \frac{M_{p}}{\Delta t} \frac{1}{V_{\text {cell }}} \delta_{z}
$$

with the phase source determined using

$$
\begin{aligned}
& \rho_{i}= \begin{cases}-\rho_{g} & \text { for gas phase } \\
+\rho_{l} & \text { for liquid source }\end{cases} \\
& \vec{S}_{M}=S_{\alpha} \vec{U}_{p}
\end{aligned}
$$

where the current computational cell volume is $V_{\text {cell }}$ in $m^{3}$ and $M_{p}$ is the mass of the particle and $\delta_{z}$ is a limiting function described by Eq. (18). The mass added in each cell, $M_{p}$, is a fraction of the total droplet mass decided by the number of cells available in the control 
volume within the scan-zone. This is needed for example, when $R_{s}>R_{w}$. A case when some of the cells in the scan-zone are outside the domain. In Fig. 3, $R_{w}$ is the radius of a sphere which has same size as the film thickness, $h_{f i l m}$, at the point of impact, $P$.

If the whole mass source is added into only the central cell (without spreading the source terms), the solution can deteriorate rapidly with large, unphysical velocities and pressures being calculated and rises in the corresponding residuals. On the other hand, experience has shown that, instability issues might be experienced if the mass is spread over too many cells too. Currently spreading over more than 20 cells is not recommended.

The proposed algorithm involves locating and marking the neighbour cells before distributing the source terms. The range of cells to scan in the "Spread Radius", $R_{s}$, can be estimated based on the DPM particle diameter, $D_{p}$, at a position, $P$ with coordinates $X_{p}$, and the cell centroids, $C$ [i] with coordinates $X_{c}$, as given in Eq. (18) based on the distances from the DPM droplet centre to the centroids of the cells in Fig. 3. No source term is added outside of the spread zone. A cell is considered to be outside the spread zone when its centroid to impact distance is greater than $0.5 D_{p}$.

$$
\delta_{z}= \begin{cases}1 & \text { if } \frac{\sqrt{\sum_{i}^{n}\left(X_{p[i]}-X_{c[i]}\right)^{2}}}{0.5 D_{p}} \leq 1 \\ 0 & \text { Out of range }\end{cases}
$$

\subsubsection{Splashing droplets as DPM}

Consider a droplet impact at a point $P_{1}$ with an impact velocity $\vec{V}_{p}$ forming a splash of droplets at a "jet length" $J_{L}$ above the free-surface, as shown in Fig. 4. Since the crown diameter is generally below $2.0 D_{p}$ and the crown height can be as up to $2.0 D_{p}$ for high $W e$ impact $^{(11)}$, it has been considered save to use $1.5 D_{p}$ for $J_{L}$ as a starting point. This is also because using very small values of $J_{L}$ has a higher chance of making the newly created "particulate" children droplets interact with a free-surface and get converted to VoF immediately. The impact normal $\vec{N}_{1}$ and the normal, $\vec{N}_{2}$ to a plane $S_{2}$ of the secondary droplets are considered parallel for simplicity. The normals $\vec{N}\left(=\vec{N}_{1}=\vec{N}_{2}\right)$ are unit vectors obtained from the gradient of the level set function, $\nabla \phi . \vec{A}$ and $\vec{B}$ are perpendicular unit vectors in the splash plane.

$$
\begin{array}{r}
\vec{N}=\|\nabla \phi\| \\
\vec{V}_{s}=\left\|\vec{V}_{p}-2\left(\vec{V}_{p} \cdot \vec{N}\right) \vec{N}\right\| \\
\vec{P}_{2}=\vec{P}_{1}+J_{L}\left(\vec{V}_{s}\right) \\
\vec{A}=\left\|\vec{V}_{s} \times \vec{N}\right\| \\
\vec{B}=\|\vec{A} \times \vec{N}\| \\
X_{i[j]}=P_{2[j]}+r \cos \left(\theta_{i}\right) A_{[j]}+r \sin \left(\theta_{i}\right) B_{[j]}
\end{array}
$$

The operators $\|$ used in Eqs. 19a-19f are used to mean compute unit vectors from the vector within. The velocity magnitude for the unit vector $\vec{V}_{s}$ is from the experimental correlations. 
Equation 19f is the parametric equation of a ring on a sphere of radius $r$ (typically the crater radius). This equation describes the coordinates of a splashing droplets number $i$ of a total of $N_{s}$ splashing droplets and $j=<1,2,3>$ is the $j^{t h}$ component of the Cartesian coordinate. The number of droplets are spread by angle $\theta_{i}$ about 0 to $2 \pi$.

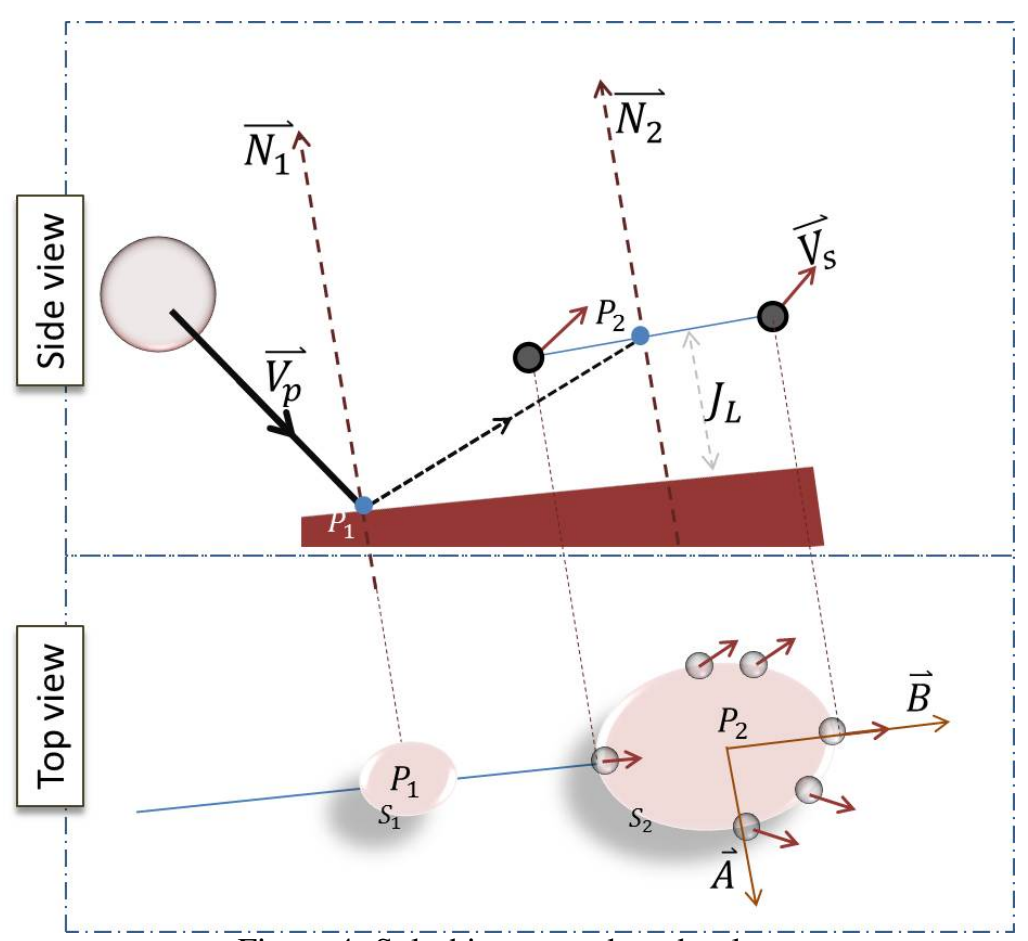

Figure 4: Splashing secondary droplets

In the review by Yarin ${ }^{(5)}$, a splash criteria is if approaching droplet velocity $U=$ $18\left\{\sigma / \rho_{l}\right\}^{\frac{1}{4}} v^{\frac{1}{8}} f^{\frac{3}{8}}<U_{o}$. It is assumed that the droplet is approaching the interface with a velocity, $U_{0}$. The kinematic viscosity is $\mu$ and the surface tension is $\sigma$. In Samenfink et al ${ }^{(33)}$, the splash criteria for film thickness in the range $0.5<h^{*}<1.8$ is when $\frac{1}{24} R e \times L a^{-0.4189}>1$. Splash criteria for thin film impact proposed in Cossali et al ${ }^{(10)}$ is that $W e \cdot O h^{-0.4}<K$, where $K=2100+5880 \cdot\left(h^{*}\right)^{1.44}$. The number of secondary droplets created by a splash for example in Okawa et al ${ }^{(28)}$, is $N_{s}=7.84 \cdot 10^{-6} \cdot K^{1.8}\left(h^{*}\right)^{-0.3}$. In crown splashing, the crown disintegrates into cusps and eventually into droplets. Rieber $\&$ Frohn ${ }^{(15)}$ correlated the number of cusps from crown splashing as a function of non-dimensional time in the form $A\left(T^{*}\right)^{-B}$, where $A$ and $B$ are constants depending on the case investigated. Other important correlations or parameters such as the velocity of exit of the droplets or the mass ejected exist in the literature $^{(16,26-28,34,35)}$. In this work, a simple presention is used. The number of droplets are evenly distributed on the parametric curve. In the implementation of the splash model, it is important to take off the mass of the children droplet from the impacting droplets before the spread of the source term. The mass of the children droplets can simply be determined from their number and mean diameters. It is worth noting that codes developed can be enhanced in the future as new and more complete splashing model correlations become available. 


\subsection{Test cases, boundary conditions and simulation setups}

There are three categories of simulations used in this work to showcase and evaluate the proposed model. These are for progressively checking (i) adequate mass conservation, (ii) momentum transfer and (iii) demonstrating splash formation using the proposed approach. Simple box type geometries with wall boundaries on all sides were used in the simulations. The dimensions of the boxes $\left(H_{x} \times H_{y} \times H_{z}\right)$ are in multiples of the droplets diameters.

\subsubsection{Mass conservation setup}

In this basic set up, a single DPM droplet is injected at the centre of an empty box. The diameter, $D_{p}$, of the droplet is $1000 \mu \mathrm{m}$. The box is a cube with a width $15 \times D_{p}$. In this test, four different mesh resolution setups are used. The meshes resolve the droplet by a spacing $S^{*}\left(=D_{p} / h\right)$ in the zones indicated on Fig. 5 with the mesh becoming coarser away from the droplet as shown; where $h$ is the grid spacing in the droplet zone. The $S^{*}$ cases tested are respectively 50, 25, 10 and 5 .

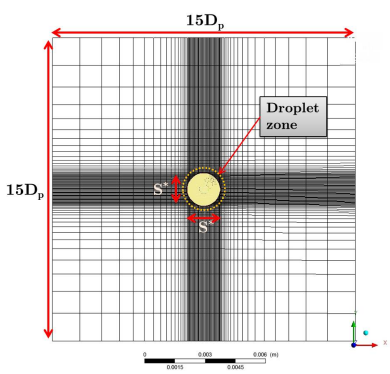

Figure 5: Mesh used for single droplet DPM-CLSVoF test case

The next basic test is for a train of DPM droplets injected into a box, as schematically shown in Fig. 6a. The flow rate is specified for the Lagrangian droplet stream and expected to fill the box to a height, $h$. Particles with a density of $1000 \mathrm{~kg} / \mathrm{m}^{3}$ are injected into an initially dry box of volume $12 \times 12 \times 20 \mathrm{~mm}^{3}$. The mesh setup is such that it is refined close to where the film is expected, Fig. $6 \mathrm{~b}$.

The number of nodes $\left(N_{x}, N_{y}, N_{z}\right)$ used for mesh dependence studies are shown in Table 1. From Fig. $6 \mathrm{~b}, N_{f}$ represents the number of nodes in the region where the film is expected. The target fill level is $1.2 \mathrm{~mm}$ for all the cases described in Table 2 . The mass of the liquid expected is $172.8 \mathrm{mg}$ in $1 \mathrm{sec}$. The droplets are spaced every $5 \times D_{p}$. In this simulation, the VoF timestep, $\Delta T$, is $1 \mu \mathrm{sec}$ to limit the Courant number to less than 1 . The convergence criteria set for all the equations are for a reduction of the residuals of 4 orders of magnitude at least for each equation. The final mass value and its stabilisation are another target.

The third is a simple extension of the previous case to test capability for multiple droplets in a domain. In this case, batches of droplets with equal diameters of $260 \mu \mathrm{m}$ are released into a box already partially filled with water. This physically represents a box being filled from a spray. Fig. 7 shows the schematic of the box, $H_{0}$ is the initial level of the film before injection starts, the filling is done by the DPM droplets within the simulation duration and fill rate such that at the end of the simulation there should be liquid in the VoF representation up to a level of $2 \times H_{0}$ by the setup in Table 3 . 


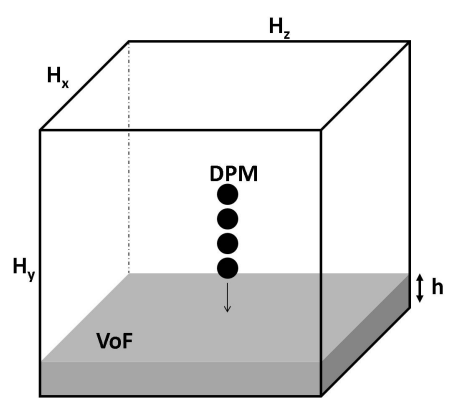

(a) Box fill setup schematic

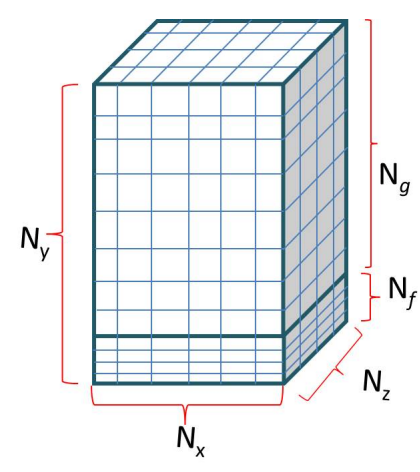

(b) Schematic of mesh setup for the box

Figure 6: DPM-CLSVoF: Simple box fill setup

Table 1: Box mesh dependence case setup

\begin{tabular}{lcclcc}
\hline \hline CASE & $N_{f}$ & Total number of nodes & $N_{x}$ & $N_{y}$ & $N_{z}$ \\
\hline$M S H_{1}$ & 15 & 14,625 & 15 & 50 & 15 \\
$M S H_{2}$ & 25 & 104,125 & 35 & 60 & 35 \\
$M S H_{3}$ & 40 & 300,000 & 50 & 80 & 50 \\
\hline
\end{tabular}
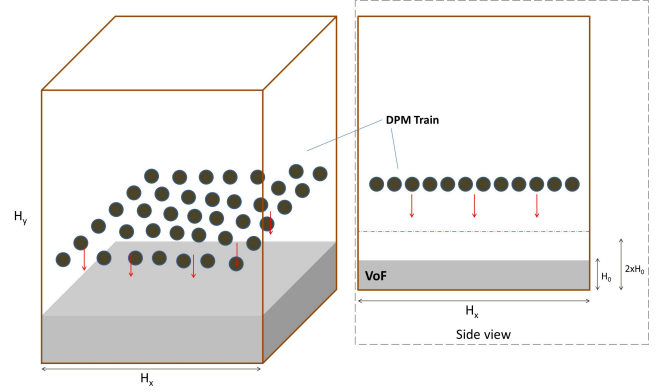

Figure 7: DPM-CLSVoF: Schematic of a Train of Multiple Droplets

\subsubsection{Demonstrating splash formation}

The impact of a droplet at a high momentum may create a good number of smaller droplets. In practical applications of droplet to film impact, the secondary droplets are equally important to account for; the film and deformation caused by the impacting droplet can also play an important role. To demonstrate splashing from a VoF film and the formation of child DPM droplets, a parent DPM droplet is injected above a VoF film. The setup follows that in the simulation work by Rieber \& Frohn ${ }^{(15)}$. The impact Weber number is 598, the Ohnesorge number, $O h$, is 0.0014 and the film thickness, $h^{*}$, is 0.116 .

There are no complete set of correlations that can completely predict all the parameters of any droplet splash scenario. The principles of operations of the model, developed here, is shown by taking several correlations from different sources in an attempt to capture the splash- 


\begin{tabular}{|c|c|c|c|c|c|}
\hline \multicolumn{2}{|c|}{ ADENIYI ET AL. } & \multicolumn{4}{|c|}{ A Coupled Euler-Lagrange CFD Modelling of Droplets-To-Film } \\
\hline \multicolumn{6}{|c|}{ Table 2: Box case setup } \\
\hline$D_{p},[\mu m]$ & $H_{x}^{*}$ & $H_{f}^{*}$ & $\begin{array}{l}\text { Number of } \\
\text { droplets }\end{array}$ & $\begin{array}{l}\text { Droplet injection fre- } \\
\text { quency, } F_{d}[\mathrm{~Hz}]\end{array}$ & DPM mass rate ${ }^{a}$ \\
\hline 150 & 80 & 8.0 & 97,785 & 1,333 & 1.77 \\
\hline 500 & 24 & 2.4 & 2,641 & 400 & 65.4 \\
\hline 800 & 15 & 1.5 & 645 & 250 & 268 \\
\hline
\end{tabular}

${ }^{a}$ The mass flow rate specified in ANSYS-Fluent GUI. This is the ratio of the mass of the dropet to the VoF timestep.

Table 3: Train of multiple droplets case setup

\begin{tabular}{ll}
\hline \hline Item & Value \\
\hline Diameter of DPM droplets, $D_{p}$ & $260 \mu \mathrm{m}$ \\
Release frequency of droplet batch, $F_{n}$ & $76.9 \mathrm{~Hz}$ \\
Droplet speed & $0.1 \mathrm{~m} / \mathrm{s}$ \\
GUI Mass rate & $920.28 \mu \mathrm{g} / \mathrm{s}$ \\
Box (square) base width, $H_{x}$ & $50 \times D_{p}$ \\
Box height, $H_{y}$ & $75 \times D_{p}$ \\
Initial film level, $H_{0}$ & $1.089 \mathrm{~mm}$ \\
Droplets spacing in a batch & $4 \times D_{p}$ \\
Height from the base with fine mesh & $3.5 \mathrm{~mm}$ \\
Number of droplets in 1 batch & $100(10 \times 10)$ \\
Total number of batches of droplets & 10 \\
\hline
\end{tabular}

ing droplets as much as possible. The number of DPM droplets generated ${ }^{(15)}$ is estimated at $T^{*}=0.1$ using the correlation $N_{s}=23.37\left(T^{*}\right)^{-0.036}$ for this case. The mean diameters of the splashing DPM droplets are estimated from the mass of splashed droplets. Equation (20a) gives the experimental correlation ${ }^{(28)}$ for the mass fraction splashed, $E_{m}$, relative to the primary droplet. By the definition of mass fraction, Eqn. (20c), and mass balance, Eqn. (20e), the diameters of the secondary droplets, $D_{s}$ can be easily obtained. The magnitude of the velocity of the splashing droplets, Eqn.(19b), at the instance of creation is $52 \%$ of the impact velocity based on the observations ${ }^{(16,28)}$ that over $80 \%$ of the splashing droplets have these speed. In this test, there are 25 splashing droplets with an average diameter $15.18 \%$ of the primary droplet.

$$
\begin{array}{r}
E_{m}=0.00156 e^{0.000486 K} \\
K=W e \cdot O h^{-2 / 5} \\
E_{m}=\frac{M_{s}}{M_{p}} \\
M_{p}=\frac{\pi}{6} \rho_{l} D_{p}^{3} \\
D_{p}^{3} E_{m}=N_{s} D_{s}^{3}
\end{array}
$$




\subsubsection{Momentum transfer setup}

The crater evolution that results from the impact of a droplet on a film can be thought of as caused by the transfer of momentum from the droplet. Normal impact with a droplet velocity of $U_{0}(\mathrm{~m} / \mathrm{s})$ are simulated. The ratio of liquid to gas density is 997 in all the cases. Other parameters are given in Table 4.

Table 4: Crater dynamics setup details.

\begin{tabular}{llllrlrll}
\hline \hline$h^{*}$ & $W e$ & Oh & $K$ & $R e$ & $U_{o}(m / s)$ & $H_{x} / D_{p}$ & $\rho_{l} / \rho_{g}$ & $\mu_{l} / \mu_{g}$ \\
\hline 0.116 & 250 & 0.00141 & 3,454 & 11,216 & 2.70 & 8.0 & 997 & 40 \\
1 & 328 & 0.00218 & 3,806 & 8,806 & 2.87 & 12.1 & 997 & 66 \\
2 & 345 & 0.00218 & 4,003 & 9,469 & 2.95 & 12.1 & 997 & 66 \\
\hline
\end{tabular}

\subsubsection{Solution method}

The solution is done in ANSYS-Fluent using the developed UDF. The second order accurate upwind discretisation is used for the spatial terms of the governing equations. The first order accurate explicit time integration is used for the temporal terms. The choice of time step is restricted with the Courant-Friedrichs-Lewy (CFL) number such that $C F L<1$. This makes simulation time step in the order of $1 \mu$ s. The first few time-steps into the solution is solved as single phase before the introduction of the DPM or the liquid phase. The DPM tracking is done transiently and the breakup models, coalescence and other schemes have been disabled to reduce complexity.

\subsection{Results}

\subsection{Mass transfer}

In converting from DPM to VoF, a sphere is expected after the conversion. The zero level set iso-surfaces in Figs. 8a to 8d show the conversions to the VoF droplets for the four levels of refinements in the single droplet test. The mesh refinement level relative to the droplet described as the ratio of droplet-size to the grid-size $\left(S^{*}=D_{p} / h\right)$. This shows a "loss in morphology" is a result of the inability to resolve the droplet to a mesh by a coarser mesh, typical of the CLSVoF method. The finer the mesh, the better the resolution of the droplet free-surface; it is, however, worth remembering that the number of cells required to resolve the droplet is actually $\left(S^{*}\right)^{3}$. The "mass loss" to poor mesh resolution can be estimated from $100\left(1-\left(R / R_{e}\right)^{3}\right)$, where $\mathrm{R}$ is the radius of the iso-surface and $R_{e}$ is the expected radius of the resulting VoF droplet.

A resolution, $S^{*}$, higher than 10 yields mass conservation over $99.6 \%$ in this single droplet test. From this simple test, a rule of thumb can be prescribed for mesh spacing in the wall regions to be of the order $S^{*}>10$; i.e. the mesh spacing to be a tenth of the mean droplet sizes, so that the first sets of DPM droplets landing on a dry wall can be resolved.

For the droplet train test, the volume of water in the geometry after the it settles down is compared with the expected volume of water $\left(17.3 \times 10^{-6} \mathrm{~m}^{3}\right)$ in the box after $1 \mathrm{sec}$. The results for Meshes $\mathrm{MSH}_{1}, \mathrm{MS} \mathrm{H}_{2}, \mathrm{MS} \mathrm{H}_{3}$ given in Table 1 are off by $8.5 \%, 1.6 \%$ and $1.49 \%$ 


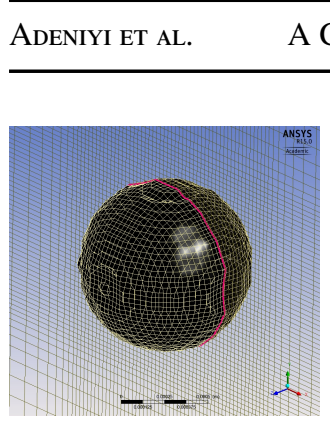

(a) $S^{*}=50$

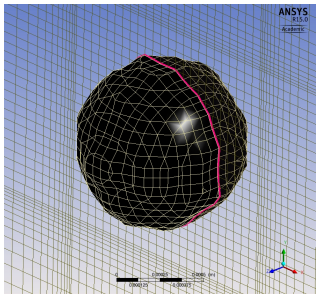

(b) $S^{*}=25$

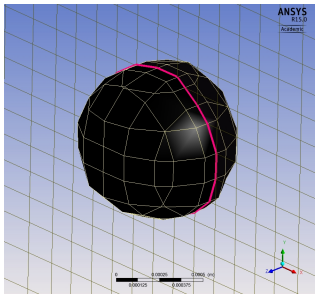

(c) $S^{*}=10$

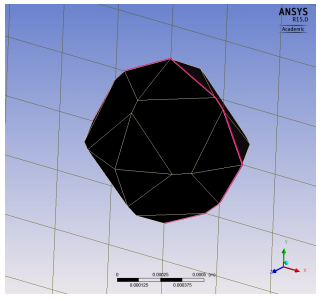

(d) $S^{*}=5$

Figure 8: Single DPM droplet turns VOF

respectively. The film grew from no-film at all to thicknesses, $H_{f}^{*}, 1.5,2.4$ and 8.0 in the 3 cases. The final error is an accumulation from the inability to resolve the first sets of landing droplets but gets better as the film develops as shown in the next test. The qualitative film formation is shown in Fig. 9 for the $800 \mu \mathrm{m}$ droplet filling the box to the marked level. The starting volume is $86.260 \times 10^{-9} \mathrm{~m}^{3}$ and the expected final volume is $172.520 \times 10^{-9} \mathrm{~m}^{3}$; the final volume computed using the finer mesh is $172.544 \times 10^{-9} \mathrm{~m}^{3}$, representing $0.013 \%$ difference; the coarser mesh with 25 nodes in the film gave a difference of $0.025 \%$.

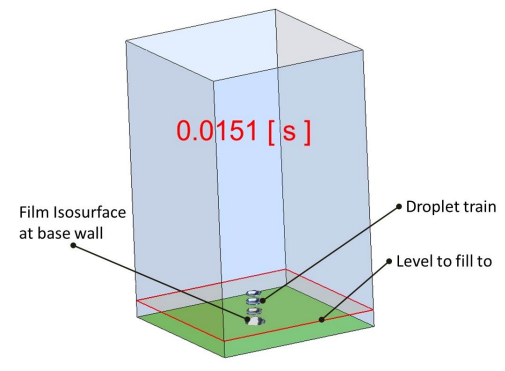

(a) The droplet train and film

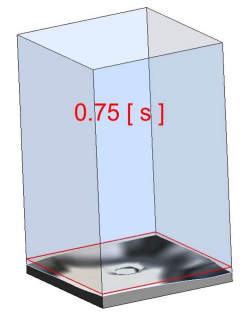

(c) $800 \mu \mathrm{m}, 0.75 \mathrm{sec}$.

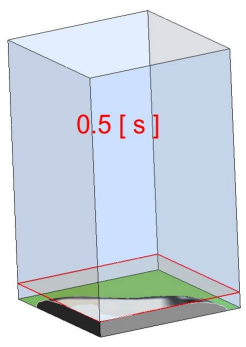

(b) $800 \mu \mathrm{m}, 0.5 \mathrm{sec}$.

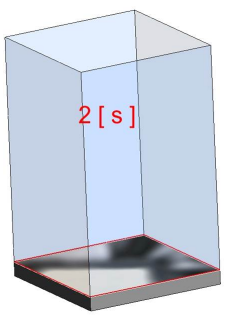

(d) $800 \mu \mathrm{m}, 2.0 \mathrm{sec}$.

Figure 9: DPM-CLSVoF: Simple box fill results $800 \mu m$ diameter droplet

Figure 10 shows the transient film formation. The two red marks show the level to fill from and to fill to. This qualitatively shows the mass conversion; with only Fig. 10c showing the droplets forming the film. 


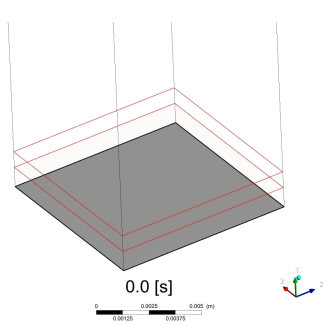

(a) Start

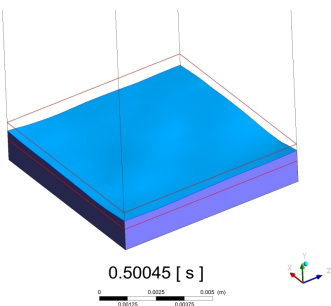

(d) $0.50045 \mathrm{~s}$

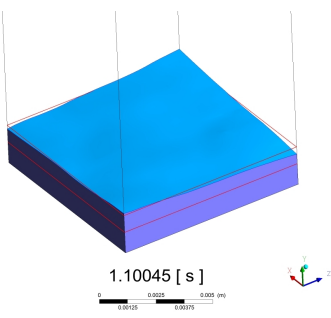

(g) $1.10045 \mathrm{~s}$

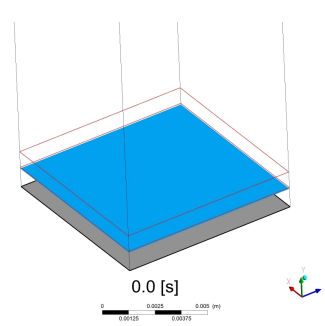

(b) Patched

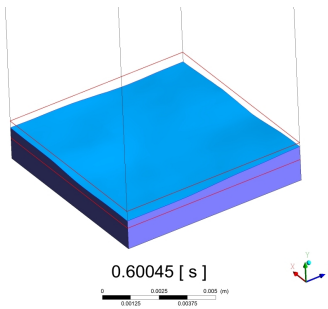

(e) $0.60045 \mathrm{~s}$

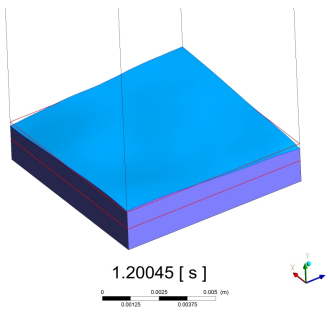

(h) $1.20045 \mathrm{~s}$

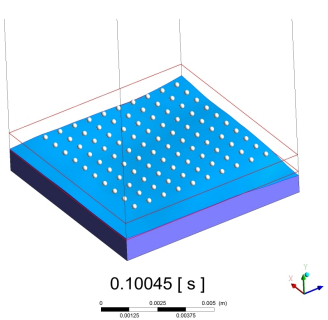

(c) $0.10045 \mathrm{~s}$

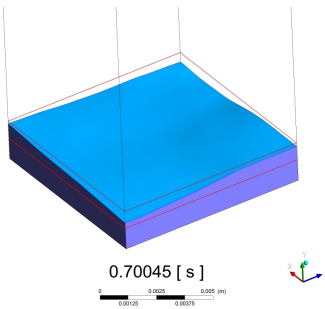

(f) $0.70045 \mathrm{~s}$

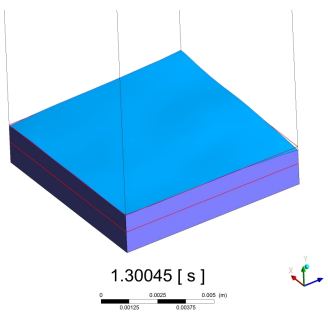

(i) $1.30045 \mathrm{~s}$

Figure 10: DPM-CLSVoF: Simple multiple droplet box fill results

\subsection{Momentum transfer}

The crater dynamics experiments for a normal impact of a single droplet on a known film thickness are presented in Fig. 11. The film thicknesses fall into thin $\left(h^{*}<1\right)$ and thick $\left(h^{*}>1\right)$ categories, with only the thin film case in the splashing category. The thin film results $\left(h^{*}=0.116\right)$, are from detailed simulations work of Rieber \& Frohn ${ }^{(15)}$, and the thick film experimental data of Cossali et $\mathrm{al}^{(11)}$, as used in Peduto et al ${ }^{(16)}$. The crown diameter, is taken as an internal diameter of the crater, which is estimated on a fixed plane coinciding with the original film surface and the impact point. A generalisation of the crown rim evolution, as suggested by Cossali et $\mathrm{al}^{(11)}$, is given in Eq. 21. The constant $C$ as proposed in Yarin \& Weiss ${ }^{(29)}$ is such that it is dependent on the initial film thickness, with $C=\left(2 / 3 h^{*}\right)^{0.25}$ and $n$ a square root form. $X$ is the crater width and the droplet diameter at impact is $D_{p}$. $T^{*}\left(=t U_{o} / D_{p}\right)$ is the non-dimensional time and $T_{o}^{*}$ is a time shifting constant.

$$
D^{*}=\frac{X}{D_{p}}=C\left(T^{*}-T_{o}^{*}\right)^{n}
$$


The experimental points are not connected with curves. The correlations, from Yarin \& Weiss ${ }^{(29)}$ and Eq. 21, are given with the dashed lines. The solid lines are results from the proposed DPM-CLSVoF simulations. It can be seen that the patterns almost exhibit a squareroot curve-fit form. It is worth noting that neither the published correlations nor the DPMCLSVoF model exactly match the experimental data points for all conditions, but follow the similar patterns. It can be seen that the thin film crater growth rate is higher than those of the thick films. The mean deviations of the simulated $D^{*}$ from the corresponding reference data points are as $4.3 \%, 15.0 \%$ and $8.1 \%$ respectively for the relative thickness values of $0.116,1$ and 2 .

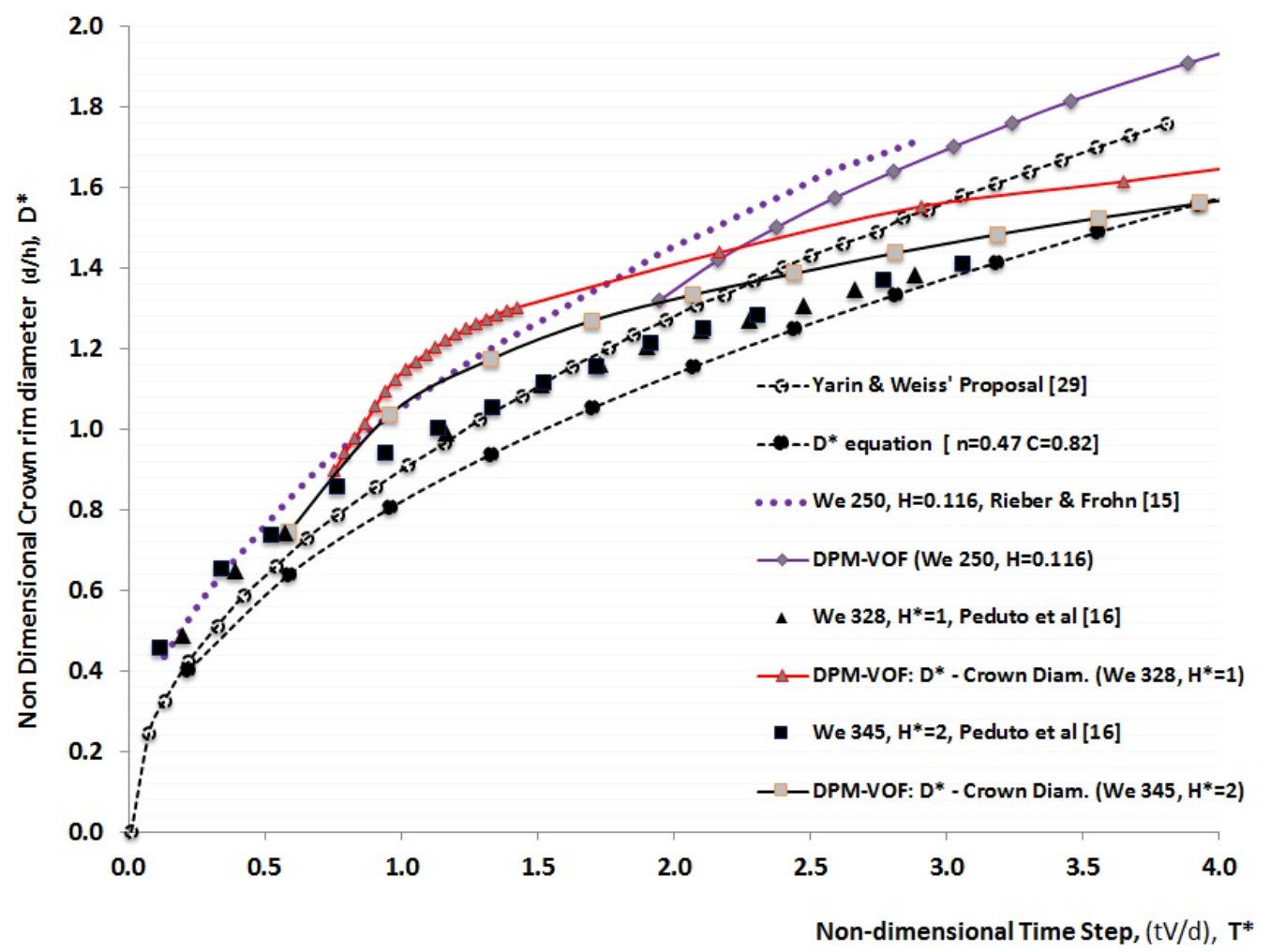

Figure 11: Crater evolution

\subsection{Splashing and creation of child droplets}

Fig. 12 shows a demonstration of a normal droplet impact as done in the detailed simulation of Rieber \& Frohn ${ }^{(15)}$. The droplet impact Weber number is 598 on the film with a thickness, $h^{*}$, of 0.116 . The DPM-CLSVoF model is computed with about 0.5 million computational cells. The DPM-CLSVoF technique is clearly unable to resolve the splashing droplets as resolved by Rieber \& Frohn ${ }^{(15)}$ who used about 32.8 million computational cells. The DPMCLSVoF technique, with that small number of cells, is not able to capture the full cusp and filament disintegration to droplets. Experimental correlations are used to predict the number 
and diameters of droplets in the splash. The otherwise "lost" droplets are created at location $A_{1}$ for continued tracking. The droplets have been shown in a time-frozen frame to give an impression of the relative locations. The primary droplet impacts at $A_{0}$ and the splashing crown starts at $A_{1}$. The crown and the secondary droplets positions grow radially outwards. The film cross section is also shown, after source term exchanges.

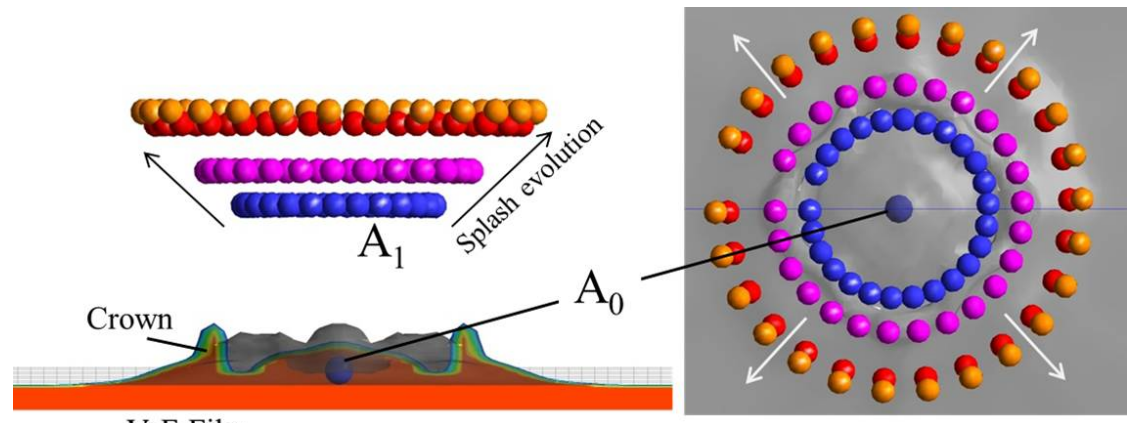

VoF Film

Figure 12: DPM-CLSVoF: Normal splashing evolution -frozen in timesteps

\subsection{Discussions}

This work provides an insight into handling droplet splash from a film as a result of a primary droplet impact. The key insight provided is that an extremely fine mesh may not be required to handle small droplets that are usually encountered in real engine droplet to film applications. There are a number of limitations to this work even though it is promising for applications requiring higher mesh resolution. It is currently limited to spherical and nondeforming droplets. Droplet breakup models and coalescence models are not included. Large droplets sizes requiring spreading source terms over a wide zone may not produce stable results at impact. Unstable results are, as expected, observed when the magnitudes of the source terms are considerably larger than the magnitude of the components of terms in the NavierStokes equations. The timestep required for solution stability is of order of 1 micro-second or lower. The DPM-CLSVoF splash simulation presented here took less than 12hours; a fully resolved splash on the same computer will take a couple of weeks to perform. The technique offers a cheap approximation to fully resolving the flow as Eulerian $\mathrm{VoF}$ and can be suited to complex geometries.

\subsection{Summary}

There has been keen interest shown by researchers on droplets to film interaction because of its wide application. Post impact outcomes of the primary droplet on a surface include, but not limited to, splashing, bouncing and sticking to the surface. The formation of film from droplet impact is useful for example in spray-film cooling. It is possible to model the detailed droplet film dynamics by fully resolving the flow with available CFD techniques. Many of these techniques, however, are computationally expensive because of the fine mesh requirements thus not practical for complex geometries. In this paper, we attempted to reduce 
the complexity involved in droplet film dynamics by assuming that the droplets can be taken to be spherical particles that can be tracked using Lagrangian technique while the liquid film and the gas phases can be taken as Eulerian using the Volume of Fluid technique. Although the coupling of Lagrangian particles with Eulerian phase is a known technique, it has not been done as it is here before. The droplets tracking takes note of the current position and the source terms are distributed to the cells. The secondary droplets are modelled from existing correlations instead of resolving them. This creates the possibility to continue to track the secondary droplets from the film with the Lagrangian technique.

The developed model was tested using simple validations for mass and momentum transfer. Several basic tests were done for mass conservation check for the Lagrangian droplets turning to Eulerian VoF film and the accuracy ranged around $0.01 \& 1.5 \%$ depending on how the film region is resolved. When a droplet impacts on a film, the consequence of momentum transfer is the crater evolution that follows it. This indirect check for momentum conservation is impressive when compared with published crater evolution results. The crater evolution simulations compare well with published correlations. For good results, the aim should be that the region where the film is most expected in the domain should be refined sufficiently to capture the film. It is not possible to be able to show detailed physics with this technique, but the solution relies on correlations to predict the impact outcome. 


\section{REFERENCES}

1. A. A. Adeniyi, H. Morvan, and K. Simmons, "A computational fluid dynamics simulation of oil-air flow between the cage and inner race of an aero-engine bearing," ASME. J. Eng. Gas Turbines Power, vol. 139, no. 1, pp. 012 506-8, 2017.

2. F. Maroteaux, D. Llory, J.-F. L. Coz, and C. Habchi, "Liquid film atomization of wall edges - separation criterion and droplets formation model." J. of Fluid Eng., vol. 124, pp. 565-575, 2002.

3. I. Owen and D. J. Ryley, "The flow of thin liquid films around corners." Int. J. of Multiph. Flow, vol. 11, no. 1, pp. 51-62, 1984.

4. J. Shinjo and A. Umemura, "Simulation of liquid jet primary breakup: Dynamics of ligament and droplet formation.” Int. J. of Multiph. Flow, vol. 36, pp. 513-532, 2010.

5. A. Yarin, "Drop impact dynamics: Splashing, spreading, receding, bouncing..." The Annu. Rev.of Fluid Mech., vol. 38, pp. 159-192, 2006.

6. A. Bisighini, "Single and double drop impacts onto a deep and thick liquid layers." Ph.D. dissertation, Universitá degli Studi di Bergamo., 2009.

7. M. Marengo, A. Carlo, R. Llia V, and T. Cameron, "Drop collisions with simple and complex surfaces." Curr. Opinion in Colloid $\mathcal{E}$ Interface Sci., vol. 16, pp. 292-302, 2011.

8. D. Brutin, "Drop impingement on a deep liquid surface: study of crater's sinking dynamics.” Comptes Rendus Mécanique, vol. 331, no. 1, pp. 61-67, 2003.

9. G. Cossali, G. Brunello, A. Coghe, and M. Marengo, "Impact of a single drop on a liquid film: Experimental analysis and comparison with empirical models." Paper presented at the Italian Congr. of Thermofluid Dyn., UIT, Ferrara., 1999.

10. G. Cossali, A. Coghe, and M. Marengo, "The impact of a single drop on a wetted solid surface." Exp. in Fluids, vol. 11, pp. 463-472, 1997.

11. G. Cossali, M. Marengo, A. Coghe, and S. Zhdanov, "The role of time in single drop splash on thin film.” Exp. in Fluids, vol. 36, no. -, pp. 888-900-, 2004.

12. S. S̆ikalo, M. Marengo, C. Tropea, and E. Ganic, "Analysis of impact of droplets on horizontal surfaces.” Exp. Thermal and Fluid Sci., vol. 25, pp. 503-510, 2002.

13. G. Cossali, M. Marengo, and M. Santini, "Multiple drop impact on heated surface." Paper presented at the 9th Int. Conf. on Liquid At. and Spray Systems, Sorrento, Italy, 2003.

14. S. S̆ikalo, C. Tropea, and E. N. Ganic, "Impact of droplets onto inclined surfaces.” J. of Colloid and Interface Sci., vol. 286, pp. 661-669, 2005.

15. M. Rieber and A. Frohn, "A numerical study on the mechanism of splashing." Int. J. of Heat and Fluid Flow, vol. 20, pp. 455-461, 1999.

16. D. Peduto, R. Koch, H. Morvan, K. Dullenkopf, and H.-J. Bauer, "Numerical studies of single drop impact onto a plane shallow and deep liquid pool." Paper presented at the ILASS -Europe 2011, 24th Ann. Conf. on Liq. Atom. and Spray Systems, Estoril, Portugal, 2011.

17. C. W. Hirt and B. D. Nichols, "Volume of fluid (vof) method for the dynamics of free boundaries.” J. of Comput. Phy., vol. 39, pp. 201-225, 1981. 
18. J. U. Brackbill, D. B. Kothe, and C. Zemach, "A continuum method for modeling surface tension.” J. of Comput. Phys., vol. 100, no. 2, pp. 335-354, 1992.

19. A. Robinson, "Computation investigation into offtake flows with application to gas turbine bearing chambers.” Ph.D. dissertation, The University of Nottingham, 2010.

20. S. Osher and J. A. Sethian, "Fronts propagating with curvature-dependent speed: algorithms based on hamilton-jacobi formulations.” J. of Comput. Phy., vol. 79, pp. 12-49, 1988.

21. M. Sussman and E. G. Puckett, "A coupled level set and volume-of-fluid method for computing $3 \mathrm{~d}$ and axisymmetric incompressible two-phase flows." J. of Comput. Phys., vol. 162, pp. 301-337, 2000.

22. J. A. Sethian and P. Smereka, "Level set methods for fluid interfaces." Annu. Rev. Fluid Mech., vol. 35, pp. 341-372, 2003.

23. G. Son and N. Hur, "A coupled level set and volume-of-fluid method for the bouyancydriven motion of fluid particles." Numer. Heat Transf., Part B: Fundamentals, vol. 42, no. 6, pp. 523-542, 2002.

24. A. Bourlioux, "A coupled level-set volume-of-fluid algorithm for tracking material interfaces." Paper presented at the Int. Symp. on Comput. Fluid Dyn., Lake Tahoe, CA., 1995.

25. P. Tkaczyk and H. P. Morvan, "Siloet: Cfd modelling guidelines of engine sumps oil and air flows simulation of bearing chambers \& sumps using an enhaced volume of fluid (vof) method," UTC in Gas Turbine Transmission Systems, The University of Nottingham, UK, Tech. Rep. JF82/PT/06, 2011.

26. C. Mundo, M. Sommerfeld, and C. Tropea, "Droplet-wall collisions: Experimental studies of the deformation and breakup process." Int. J. of Multiph. Flow, vol. 21, no. 2, pp. 151-173, 1995.

27. P. J. O'Rourke and A. A. Amsden, “A spray/wall interaction submodel for the kiva-3 wall film model.” SAE TECHNICAL PAPER SERIES 2000-01-0271., 1996.

28. T. Okawa, T. Shiraishi, and T. Mori, "Effect of impingement angle $\mathrm{n}$ the outcome of single water drop impact onto a plane water surface." Exp. Fluids, vol. 44, pp. 331-339, 2008.

29. A. L. Yarin and D. A. Weiss, "Impact of drops on solid surfaces: self-similar capillary waves, and splashing as a new type of kinematic discontinuity." J. of Fluid Mech., vol. 283, pp. 141-173, 1995.

30. ANSYS, Fluent 14.5-User Manual. $\quad$ ANSYS Inc., 2013.

31. L. Schiller and Z. Naumann, Z. Ver. Deustsch. Ing., vol. 77, p. 318, 1935.

32. A. K. W. Cheung, B. T. Tan, K. Hourigan, and M. C. Thompson, "Interference drag between spherical and cylinderical particles in stokes flow." Paper presented at the 14th Australasian Fluid Mech. Conf., Adelaide University, Australia., 2001.

33. W. Samenfink, A. Elsaßer, Dullenkopf., and S. Wittig, "Droplet interaction with sheardriven liquid films: analysis of deposition and secondary droplet characteristics." Int. J. of Heat and Fluid Flow, vol. 20, pp. 462-469, 1999.

34. C. Bai and A. D. Gosman, "Mathematical modelling of wall films formed by impinging sprays.” SAE TECHNICAL PAPER SERIES 960626, 1996. 
35. Z. Mehdizadeh, Navid, S. Chandra, and J. Mostaghimi, "Formation of fingers around the edges of a drop hitting a metal plate with high velocity," J. Fluid Mech., vol. 510, pp. 353-373, 2004. 